\title{
A FINITE DIFFERENCE DOMAIN DECOMPOSITION ALGORITHM FOR NUMERICAL SOLUTION OF THE HEAT EQUATION
}

\author{
CLINT N. DAWSON, QIANG DU, AND TODD F. DUPONT
}

\begin{abstract}
A domain decomposition algorithm for numerically solving the heat equation in one and two space dimensions is presented. In this procedure, interface values between subdomains are found by an explicit finite difference formula. Once these values are calculated, interior values are determined by backward differencing in time. A natural extension of this method allows for the use of different time steps in different subdomains. Maximum norm error estimates for these procedures are derived, which demonstrate that the error incurred at the interfaces is higher order in the discretization parameters.
\end{abstract}

\section{INTRODUCTION}

There are two motivations for the use of domain decomposition in the processes defined and analyzed here. First, domain decomposition is a natural way to develop methods for numerically approximating solutions to partial differential equations on parallel computers. Second, even on sequential computers, it is useful to be able to use different time steps on different subdomains.

A natural way to solve partial differential equations in parallel is to divide the domain over which the problem is defined into subdomains, and solve the subdomain problems in parallel. The major difficulties with such procedures involve defining values on the subdomain boundaries and piecing the solutions together into a reasonable approximation to the true solution. It is almost a side effect of dividing the problem into subproblems that one can approximate the parts of the solution with greater independence, and this leads naturally to methods that allow different time steps on different subdomains.

Much of the work on domain decomposition has been directed at elliptic equations (see, for example, $[1,2,4,6]$ and the references therein). Such algorithms could quite easily be applied to parabolic equations, giving domain decomposition iterative methods for the solution of the equations at each time step. Another approach has been given in [5] and adapted in [7, 8]; these

Received December 7, 1989; revised August 3, 1990.

1980 Mathematics Subject Classification (1985 Revision). Primary 65N10, 65P05.

Key words and phrases. Domain decomposition, parabolic equations, finite differences, parallel computing, spatially varying time step.

The first author was supported under National Science Foundation Grant No. DMS-8807257. 
methods use overlapping subdomains to approximately solve the implicit equations arising from a standard finite difference discretization.

In this paper, we present a finite difference method which utilizes domain decomposition to allow us to divide the work of solving the heat equation. The method differs from the methods mentioned above in that it uses nonoverlapping subdomains and is noniterative. Our purpose here is to introduce and analyze a fairly simple algorithm in one and two space dimensions. We restrict attention to intervals and squares, and derive maximum norm error estimates. In a separate article [3], two of the authors develop Galerkin formulations of analogous algorithms, and give energy-norm error estimates.

The rest of this paper is organized as follows. In the next section, we define a domain decomposition process for a one-space-dimensional problem and prove a convergence result for it. An interesting aspect of the error bound is that the error associated with the interface between the subdomains is higher order in the discretization parameters; this reflects the fact that this truncation error is confined to a small set. In $\S 3$, we give a straightforward generalization of the one-dimensional results to two space dimensions, state an error bound, and remark on further extensions.

\section{ONE-SPACE-DIMENSIONAL DOMAIN DECOMPOSITION}

Let $u(x, t)$ be the solution of the heat equation

$$
\begin{gathered}
\frac{\partial u}{\partial t}-\frac{\partial^{2} u}{\partial x^{2}}=0, \quad x \in(0,1), \quad t \in(0, T], \\
u(x, 0)=u^{0}(x), \quad x \in(0,1), \\
u(0, t)=u(1, t)=0, \quad t \in(0, T] .
\end{gathered}
$$

2.1. Basic 1-d method. For simplicity, we will consider first a numerical method which involves decomposing $(0,1)$ into only two subdomains, $(0, \bar{x})$ and $(\bar{x}, 1)$.

For a positive integer $N$, let $h=1 / N$, and take $x_{i}=i h, i=0, \ldots, N$. Assume that $\bar{x}$ and $N$ are such that $\bar{x}=x_{K}>0$ for some integer $K$. A related parameter is $H>0$, which is an integral multiple of $h$ and does not exceed $\min (\bar{x}, 1-\bar{x})$. Take $\Delta t=T / M$, where $M$ is a positive integer, and let $t^{n}=n \Delta t$. For a function $f(x, t)$ defined at mesh points $\left(x_{i}, t^{n}\right)$, let $f_{i}^{n}=f\left(x_{i}, t^{n}\right)$. Define the difference operators

$$
\partial_{t, \Delta t} f(t)=\frac{f(t)-f(t-\Delta t)}{\Delta t}
$$

and

$$
\partial_{x, h}^{2} f(x)=\frac{f(x-h)-2 f(x)+f(x+h)}{h^{2}} .
$$

We will refer to points $\left(x_{i}, t^{n}\right)$ as boundary points if $i=0$ or $N$, or if $n=0$. Similarly, we refer to them as interface points if $x_{i}=\bar{x}$ and $n>0$. Otherwise, they are interior points. 
The numerical approximation $U_{i}^{n}$ to $u_{i}^{n}$ is defined by

$$
\begin{array}{rlrl}
U_{i}^{n} & =u_{i}^{n} & & \text { at boundary points, } \\
\partial_{t, \Delta t} U_{i}^{n}-\partial_{x, H}^{2} U_{i}^{n-1} & =0 & & \text { at interface points, } \\
\partial_{t, \Delta t} U_{i}^{n}-\partial_{x, h}^{2} U_{i}^{n}=0 & & \text { at interior points. }
\end{array}
$$

This numerical approximation is given by an explicit forward difference formula on the interface, while in the interior of the subdomains it satisfies an implicit backward difference equation. One would expect that there will be a constraint of the form

$$
\Delta t \leq \frac{1}{2} H^{2}
$$

but that $\Delta t$ will not be constrained by the size of $h$; this turns out to be correct. Notice that in advancing the solution from time level $t=t^{n-1}$ to $t=t^{n}$ one first computes the value of $U$ at the interface. This step requires a small amount of information from each subdomain. After the interface value has been computed, there are two completely separate backward difference problems to solve, which can be done in parallel.

The approximate solution $U$ satisfies the following a priori error estimate:

Theorem 1. Suppose that $\frac{1}{2}\left|\partial^{2} u / \partial t^{2}\right|$ and $\frac{1}{12}\left|\partial^{4} u / \partial x^{4}\right|$ are bounded by $C_{0}$ on $[0,1] \times[0, T]$. Suppose also that $\Delta t \leq H^{2} / 2$. Then

$$
\max _{i, n}\left|u\left(x_{i}, t^{n}\right)-U_{i}^{n}\right| \leq \frac{C_{0}}{4}\left(h^{2}+H^{3}+\Delta t\right) .
$$

Since the difference operators used to define $U$ are second-order-correct in space and first-order-correct in time, the result is perhaps surprising only because of the presence of the $H^{3}$ term.

Asymptotically one would expect to choose $\Delta t, h$, and $H$ so as to balance the terms in (10); i.e., $\Delta t \approx h^{2} \approx H^{3}$. If such choices are made, then $\Delta t=o\left(H^{2}\right)$ and (9) is automatically satisfied in the range of small errors.

The proof of Theorem 1 relies on the following maximum principle.

Lemma 1. Suppose that $\Delta t \leq H^{2} / 2$ and that $z_{i}^{n}$ satisfies the following relations:

$$
\begin{aligned}
z_{i}^{n} & \leq 0 \text { at boundary points, } \\
\partial_{t, \Delta t} z_{i}^{n}-\partial_{x, H}^{2} z_{i}^{n-1} & \leq 0 \text { at interface points, }
\end{aligned}
$$$$
\partial_{t, \Delta t} z_{i}^{n}-\partial_{x, h}^{2} z_{i}^{n} \leq 0 \text { at interior points. }
$$

Then, for each $i$ and $n$,

$$
z_{i}^{n} \leq 0
$$

Proof of Lemma 1. Note that (14) holds for $n=0$. Now suppose the conclusion holds up to some level $n-1$. Then the interface value $z_{K}^{n} \leq 0$; it is bounded above by an average of values of $z_{i}^{n-1}$, and the weights in the average are 
nonnegative because of the constraint on $\Delta t$. This observation is, of course, just the usual one used to prove the maximum principle for the explicit difference formula for the heat equation.

Next, $z_{i}^{n} \leq 0$ at interior points by the maximum principle for the backward difference equation applied to the two subdomains. Note that this does not require that $\Delta t$ be constrained in any way based on the size of $h$.

Proof of Theorem 1. Let $e_{i}^{n}=u_{i}^{n}-U_{i}^{n}$. Then

$$
\begin{aligned}
e_{i}^{n}=0 & \text { at boundary points, } \\
\partial_{t, \Delta t} e_{i}^{n}-\partial_{x, H}^{2} e_{i}^{n-1}=K_{i}^{n}\left(\Delta t+H^{2}\right) & \text { at interface points, }
\end{aligned}
$$$$
\partial_{t, \Delta t} e_{i}^{n}-\partial_{x, h}^{2} e_{i}^{n}=K_{i}^{n}\left(\Delta t+h^{2}\right) \text { at interior points, }
$$

where

$$
\left|K_{i}^{n}\right| \leq C_{0} .
$$

Let $\theta_{0}=\theta_{N}=0$, and suppose that for $0<i<N$,

$$
-\partial_{x, h}^{2} \theta_{i}=1 \text {. }
$$

Then

$$
\theta_{i}=\frac{1}{2} x_{i}\left(1-x_{i}\right)
$$

and, in particular,

$$
0 \leq \theta_{i} \leq \frac{1}{8} .
$$

Choose $\beta_{i}, i=0, \ldots, N$, to satisfy $\beta_{0}=\beta_{N}=0$ and

$$
\begin{gathered}
\partial_{x, h}^{2} \beta_{i}=0, \quad 0<i<N, \quad i \neq K, \\
-\partial_{x, H} \beta_{K}=1 .
\end{gathered}
$$

Then

$$
\beta_{i}= \begin{cases}H x_{i}(1-\bar{x}), & 0 \leq x_{i} \leq x_{K}=\bar{x} \\ H\left(1-x_{i}\right) \bar{x}, & x_{K} \leq x_{i} \leq 1\end{cases}
$$

hence,

$$
0 \leq \beta_{i} \leq H / 4 \text {. }
$$

Let

$$
\xi_{i}=C_{0}\left[\theta_{i}\left(\Delta t+h^{2}\right)+\beta_{i}\left(\Delta t+H^{2}\right)\right],
$$

and set

$$
z_{i}^{n}=e_{i}^{n}-\xi_{i} .
$$

Since $z_{i}^{n}$ satisfies the conditions of Lemma 1 , we see that $z_{i}^{n} \leq 0$; hence,

$$
e_{i}^{n} \leq \xi_{i}
$$


Similarly, by taking $z_{i}^{n}=-e_{i}^{n}-\xi_{i}$, we see that $-e_{i}^{n}$ is aiso bounded above by $\xi_{i}$. Hence we conclude that

$$
\left|e_{i}^{n}\right| \leq \xi_{i} .
$$

Finally, by the construction of $\xi_{i}$ we see that

$$
\begin{aligned}
0 & \leq \xi_{i} \leq C_{0}\left[\frac{1}{8}\left(\Delta t+h^{2}\right)+\frac{1}{4} H\left(\Delta t+H^{2}\right)\right] \\
& \leq \frac{1}{4} C_{0}\left(h^{2}+\Delta t+H^{3}\right) .
\end{aligned}
$$

Note that $\bar{x}$ can vary with $N$, since the exact value of $\bar{x}$ does not change the error bound.

2.2. Spatially varying time and space steps. Now suppose that $\Delta t_{I}>0$ is a time step associated with the interface and that $\Delta t_{L}$ and $\Delta t_{R}$ are obtained from $\Delta t_{I}$ by dividing it by two, possibly different, positive integers, $m_{L}$ and $m_{R}$. To advance the solution from $(n-1) \Delta t_{I}$ to $n \Delta t_{I}$, first take an explicit step of the form (7) using $\Delta t_{I}$ and $H$. Then on the left and right subdomains take $m_{L}$ and $m_{R}$ steps of the backward difference equation. The boundary values on the interface at intermediate times are obtained by linear interpolation of the interface values.

We can use different $h$ 's on the left and right of $\bar{x}$, but the $H$ for the interface will be assumed to be an integral multiple of each. Denote by $h_{L}$ and $h_{R}$ the space steps in the left and right subdomains, respectively. If we let $C_{0 L}$ and $C_{0 R}$ be bounds for $\frac{1}{2}\left|\partial^{2} u / \partial t^{2}\right|$ and $\frac{1}{12}\left|\partial^{4} u / \partial x^{4}\right|$ on the left and right regions and let $C_{I}$ be a similar bound for $|x-\bar{x}| \leq H$, then we can get an error bound analogous to that in Theorem 1.

Specifically, define

$$
\begin{aligned}
\xi(x)= & C_{0 L} \theta_{L}(x)\left(\Delta t_{L}+h_{L}^{2}\right)+C_{0 R} \theta_{R}(x)\left(\Delta t_{R}+h_{R}^{2}\right) \\
& +C_{I} \beta(x)\left(\Delta t_{I}+H^{2}\right),
\end{aligned}
$$

where $\theta_{L}$ is a continuously differentiable function, defined by $\theta_{L}(0)=\theta_{L}(1)=$ 0 and

$$
-\theta_{L}^{\prime \prime}(x)= \begin{cases}1, & 0 \leq x<\bar{x} \\ 0, & \bar{x} \leq x<1\end{cases}
$$

$\theta_{R}$ is defined analogously, and $\beta(x)$ satisfies (24). Note that $\theta_{L}$ and $\theta_{R}$ are nonnegative functions whose sum is bounded by $\frac{1}{2} x(1-x) \leq \frac{1}{8}$. Then, by an argument that is very similar to the proof of Theorem 1 we see that

$$
\left|U_{i}^{n}-u_{i}^{n}\right| \leq \xi_{i}=\mathscr{O}\left(h_{L}^{2}+h_{R}^{2}+H^{3}+\Delta t_{R}+\Delta t_{L}+H \Delta t_{I}\right) .
$$

One point in the proof of (33) that we found to be subtle is the way interface values at intermediate times are viewed. Computationally, the recipe was given 
in the first paragraph of this section; advance the solution by $\Delta t_{I}$ and linearly interpolate. However, if one involves the interpolant in time of the true solution $u$ in the analysis, there are problems with the truncation error at interior points next to the interface. The solution to this difficulty is very simple: one views the intermediate-time interface values as having been computed by a first-ordercorrect explicit scheme. The maximum principle used is stated as Lemma 2; its proof is very similar to that of Lemma 1.

Lemma 2. Suppose that $z$ is a grid function defined on the $\left(h_{L}, \Delta t_{L}\right)$ and $\left(h_{R}, \Delta t_{R}\right)$ grids of the left and right regions, respectively, including those grid points on the boundary and interface. Suppose that $z \leq 0$ at all boundary points, and that $\partial_{t, \Delta t} z-\partial_{x, h}^{2} z \leq 0$ at all interior points, with $\Delta t=\Delta t_{L}$ or $\Delta t_{R}$ and $h=h_{L}$ or $h_{R}$, as appropriate. Also suppose that for $t=n \Delta t_{I}$ and $1 \leq m \leq m_{L}$,

$$
\frac{z\left(\bar{x}, t+m \Delta t_{L}\right)-z(\bar{x}, t)}{m \Delta t_{L}}-\partial_{x, H}^{2} z(\bar{x}, t) \leq 0
$$

suppose the analogous inequality holds for $m \Delta t_{R}$ as well. Then $z \leq 0$ at each grid point, provided $\Delta t_{I} \leq H^{2} / 2$.

Note that the approximate solution $U$ satisfies (34), with inequality replaced by equality, when the linear interpolant is used to define values at $t+m \Delta t_{L}$, $m=1, \ldots, m_{L}$.

2.3. Many subdomains. In $\S \S 2.1$ and 2.2 , we used only two subdomains; however, the arguments employed are sufficiently simple that they can easily be extended to the case of multiple subdomains. Suppose that we use a single time step and a uniform mesh spacing $h$ as in $\S 2.1$. Suppose that

$$
0<H \leq \bar{x}_{1}<\bar{x}_{2}<\cdots<\bar{x}_{J} \leq 1-H
$$

are all multiples of $h$; that is, $\bar{x}_{l}=j h=x_{j}$ for some $j$. Take $U_{j}^{n}$ to be defined as in $\S 1$ except that at each point $\bar{x}_{l}$ we use an explicit difference formula for $U$ at the advanced time level. It is clear that the analogue of Lemma 1 remains valid. This gives a bound very similar to that in Theorem 1. Specifically, with $\Delta t \leq H^{2} / 2$ and $C_{0}$ as in Theorem 1 ,

$$
\max _{i, n}\left|u_{i}^{n}-U_{i}^{n}\right| \leq \frac{C_{0}}{8}\left[h^{2}+\Delta t+2 J H\left(H^{2}+\Delta t\right)\right]
$$

Unless $J H$ is small, the interface error dominates this expression; but when only a few subdomains are used (that is, $J$ is small), the interface error can be quite small. 


\section{TWO-SPACE-DIMENSIONAL DOMAIN DECOMPOSITION}

In this section, $u(x, y, t)$ will be a solution of the heat equation on $\Omega=$ $(0,1) \times(0,1)$. Specifically, $u$ satisfies

$$
\begin{gathered}
\frac{\partial u}{\partial t}-\Delta u=0, \quad(x, y) \in \Omega, t \in(0, T], \\
u(x, 0)=u^{0}(x), \quad(x, y) \in \Omega, \\
u(x, t)=0, \quad(x, y) \in \partial \Omega .
\end{gathered}
$$

Here, $\Delta u=\partial^{2} u / \partial x^{2}+\partial^{2} u / \partial y^{2}$.

3.1. A basic 2-d method. We start with a simple two-domain scheme. Take

$$
\Omega_{1}=\{(x, y) \in \Omega: x<\bar{x}\}, \quad \Omega_{2}=\{(x, y) \in \Omega: \bar{x}<x\} .
$$

Let $x_{i}=i h$ just as in $\S 2$, and let $y_{j}=j h, j=0, \ldots, N$. Suppose that $\bar{x}$ and $H$ are integral multiples of $h$, that $0<\bar{x}<1$, and that $0<H \leq \min (\bar{x}, 1-\bar{x})$. In analogy with $\S 2$ we will call a point $\left(x_{i}, y_{j}, t^{n}\right)$ a boundary point if $n=0$ or if $\left(x_{i}, y_{j}\right) \in \partial \Omega$. Such a point with $x_{i}=\bar{x}$ will be an interface point if it is not a boundary point. The remaining points $\left(x_{i}, y_{j}, t^{n}\right)$ are in $\left(\Omega_{1} \cup \Omega_{2}\right) \times(0, T]$ and are interior points.

The values $U_{i j}^{n}$ will approximate $u_{i j}^{n}=u\left(x_{i}, y_{j}, t^{n}\right)$. With the natural extension of the notation of (4) and (5), we define $U_{i j}^{n}$ by the following relations:

$$
\begin{aligned}
U_{i j}^{n} & =u_{i j}^{n} & & \text { at boundary points, } \\
\partial_{t, \Delta t} U_{i j}^{n}-\partial_{x, H}^{2} U_{i j}^{n-1}-\partial_{y, h}^{2} U_{i j}^{n} & =0 & & \text { at interface points, } \\
\partial_{t, \Delta t} U_{i j}^{n}-\partial_{x, h}^{2} U_{i j}^{n}-\partial_{y, h}^{2} U_{i j}^{n} & =0 & & \text { at interior points. }
\end{aligned}
$$

Notice that the computation of $U$ along the interface $x=\bar{x}$ requires the solution of a tridiagonal set of equations; this is a small amount of work when compared to the work to solve the subdomain problems (42). The two subdomain problems (42) are entirely separate, once the values on the interface have been computed. If we use the techniques of the proof of Theorem 1, we can derive an error estimate of the following form.

Theorem 2. Suppose that

$$
\frac{1}{2}\left|\partial^{2} u / \partial t^{2}\right|,\left|\partial^{3} u / \partial t \partial x^{2}\right|, \quad \text { and } \frac{1}{12}\left[\left|\partial^{4} u / \partial x^{4}\right|+\left|\partial^{4} u / \partial y^{4}\right|\right]
$$

are bounded by $C_{0}$. Suppose also that $\Delta t \leq H^{2} / 2$. Then

$$
\max _{i, j, n}\left|u_{i j}^{n}-U_{i j}^{n}\right| \leq \frac{C_{0}}{8}\left[h^{2}+\Delta t+2 H\left(2 \Delta t+H^{2}\right)\right] .
$$

A proof of this theorem uses the analogue of Lemma 1 and even uses exactly the same one-dimensional functions $\theta$ and $\beta$ as in $\S 2.1$. The cross-derivative 
term comes into the error bound because the spatial operator on the interface is evaluated at two different time levels.

3.2. Spatially varying time steps. In this subsection, we consider the effect of using different time steps on the interface and the left and right subdomains. We do not discuss the changes that come about from changing the spatial mesh.

Take $\Delta t_{I}, \Delta t_{L}$, and $\Delta t_{R}$ as in $\S 2.2$; that is, for positive integers $m_{L}$ and $m_{R}$, $0<\Delta t_{I}=m_{L} \Delta t_{L}=m_{R} \Delta t_{R}$. To advance the solution from $(n-1) \Delta t_{I}$ to $n \Delta t_{I}$, first compute the interface values at the new time by solving the tridiagonal system (41), and interpolate linearly in time to obtain the interface values at the intermediate times. These interface values then decouple the subdomain problems; using them, one takes $m_{L}$ or $m_{R}$ steps to advance the solution at the interior points.

Under the hypotheses of Theorem 2, one can prove the following error bound:

$$
\max _{i, j, n}\left|U_{i j}^{n}-u_{i j}^{n}\right|=\mathscr{O}\left(h^{2}+H^{3}+\Delta t_{L}+\Delta t_{R}+H \Delta t_{I}\right) \text {. }
$$

Here, as in $\S 2.2$, the way that the intermediate-time interface points are viewed is important. The analogue of (34) in this context is (with $\Delta t=m \Delta t_{L}$, $\left.y=j h, t=n \Delta t_{I}, 0<m \leq m_{L}\right)$

$$
\partial_{t, \Delta t} z(\bar{x}, y, t+\Delta t)-\partial_{x, H}^{2} z(\bar{x}, y, t)-\partial_{y, h}^{2} \tilde{z}_{m}(\bar{x}, y) \leq 0,
$$

where

$$
\tilde{z}_{m}(\bar{x}, y)=z(\bar{x}, y, t)+\frac{m_{L}}{m}(z(\bar{x}, y, t+\Delta t)-z(\bar{x}, y, t)) .
$$

Note that $U(\bar{x}, y, t)$ satisfies (45) with equality instead of inequality, and $u$ satisfies (45) up to a truncation error which is $\mathscr{O}\left(\Delta t+H^{2}+h^{2}\right)$.

In $\S 2.2$, the relation (34) made it immediately clear that if all the $z$ 's at time level $t=n \Delta t_{I}$ were nonpositive, then all the interface values at times $t+m \Delta t_{L}$ were also nonpositive. However, (45) does not have the property that it gives $z(\bar{x}, y, t+\Delta t)$ as a nonnegatively weighted average of the other values involved. A way to see that $z(\bar{x}, y, t+\Delta t) \leq 0$, provided $z \leq 0$ at $t=n \Delta t_{I}$ and appropriate boundary conditions hold, is to extrapolate the change in $z$ out to $t+\Delta t_{I}$ and look at the difference equation satisfied by the extrapolated function. One can conclude that even after extrapolation the function is still nonpositive, and that implies that $z(\bar{x}, y, t+\Delta t)$ is nonpositive. Specifically, (46) and (45) give

$$
\frac{\tilde{z}_{m}(\bar{x}, y)-z(\bar{x}, y, t)}{\Delta t_{I}}-\partial_{x, H}^{2} z(\bar{x}, y, t)-\partial_{y, h}^{2} \tilde{z}_{m}(\bar{x}, y) \leq 0 .
$$

Since this relation gives an upper bound for $\tilde{z}_{m}(\bar{x}, y)$ that is a nonnegatively weighted average of the other values involved, it is easy to see that $\tilde{z}_{m}(\bar{x}, y)$ $\leq 0$, provided that $z(\bar{x}, y, t) \leq 0$ and the boundary values $\tilde{z}_{m}(\bar{x}, 0)$ and $\tilde{z}_{m}(\bar{x}, 1)$ are nonpositive. To assure that the boundary values are nonpositive, we need more than the fact that $z \leq 0$ at boundary points. In our application of the maximum principle, $z=-\xi \pm(u-U)$, a function which is both nonpositive 
on the boundary and independent of time at each boundary point. Thus, we conclude that $\tilde{z}_{m}(\bar{x}, y) \leq 0$, and in particular, this holds for $m=m_{L}$, which gives the desired result.

3.3. Remarks on extensions. It is straightforward to extend the two-dimensional results to allow for many subdomains, at least if one restricts attention to the case of dividing the square into vertical strips. The error estimate is very similar to that discussed in $\S 2.3$, but it needs to be modified just as Theorem 1 was modified to get Theorem 2 .

The procedure and the error estimate of $\S 3.1$ is clearly generalizable to $n$ dimensional space. The interface problem now involves the solution of an "elliptic" equation on an $(n-1)$-dimensional domain.

\section{BIBLIOGRAPHY}

1. P. E. Bjørstad and O. B. Widlund, Iterative methods for the solution of elliptic problems on regions partitioned into substructures, SIAM J. Numer. Anal. 23 (1986), 1097-1120.

2. J. H. Bramble, J. E. Pasciak, and A. H. Schatz, The construction of preconditioners for elliptic problems by substructuring. I, Math. Comp. 47 (1986), 103-134.

3. C. N. Dawson and Q. Du, A finite element domain decomposition method for parabolic equations, Rice Technical Report TR90-25, Dept. of Math. Sciences, Rice University.

4. R. Glowinski and M. F. Wheeler, Domain decomposition and mixed finite element methods for elliptic problems, Proc. First Internat. Sympos. on Domain Decomposition Methods for Partial Differential Equations, SIAM, Philadelphia, PA, 1988.

5. Y. Kuznetsov, New algorithms for approximate realization of implicit difference schemes, Soviet J. Numer. Anal. Math. Modelling 3 (1988).

6. P. L. Lions, On the Schwarz alternation method. I, Proc. First Internat. Sympos. on Domain Decomposition Methods for Partial Differential Equations, SIAM, Philadelphia, PA, 1988.

7. G. Meurant, Numerical experiments with a domain decomposition method for parabolic problems, Proc. Fourth Internat. Sympos. on Domain Decomposition Methods for Partial Differential Equations, Moscow, May 1990 (to appear).

8. __ A domain decomposition method for parabolic equations, Appl. Numer. Math. (to appear).

(C. N. Dawson and Qiang Du) Department of Mathematics, University of Chicago, Chicago, Illinois 60637

Current address, C. N. Dawson: Department of Mathematical Sciences, Rice University, Houston, Texas 77251

E-mail address: C. N. Dawson: clint@rice.edu

Current address, Q. Du: Department of Mathematics, Michigan State University, Lansing, Michigan 48824

(T. F. Dupont) Department of Computer Science, University of Chicago, Chicago, IlliNoIs 60637 